International Mathematical Forum, 2, 2007, no. 62, 3079 - 3084

\title{
On Minimal Projective Resolution
}

\author{
M. A. Al Shumrani \\ Department of Mathematics, King Abdulaziz University \\ P.O. Box: 80203 Jeddah 21589, Kingdom of Saudi Arabia \\ maalshmrani1@kau.edu.sa
}

\begin{abstract}
The aim of this paper is to prove that for any unbounded chain complex $Y$ in the category $\mathbf{C h}(R)$ of chain complexes whose homology modules $H_{i}(Y)$ are of finite type has a minimal projective resolution where $R$ is a commutative hereditary local ring.
\end{abstract}

\section{Mathematics Subject Classification: 55U15}

Keywords: minimal projective resolution, category of chain complexes

\section{Introduction}

Let $R$ be a commutative hereditary local ring with maximal ideal $\mathfrak{m}$ and residue field $\mathbb{K}=R / \mathfrak{m}$.

Now we recall some basic definitions.

Definition 1.1. A chain map $f: X \longrightarrow Y$ is called a q-isomorphism if the maps $f_{\star}: H_{n}(X) \longrightarrow H_{n}(Y)$ are all isomorphisms.

Definition 1.2. A projective resolution of a chain complex $Y$ is a q-isomorphism $P \longrightarrow Y$ such that each $P_{i}$ is a projective $R$-module.

Definition 1.3. A chain complex $(Y, d)$ is minimal if the induced differential $d \otimes \operatorname{id}_{\mathbb{K}}$ on $Y \otimes \mathbb{K}[0]$ satisfies $d \otimes \operatorname{id}_{\mathbb{K}}=0$.

We note that for a chain complex $Y$ if $d\left(Y_{n}\right) \subseteq \mathfrak{m} Y_{n-1}$, then $d \otimes \mathrm{id}_{\mathbb{K}}=0$.

The following result is important and will play a major role in proving the minimal part of the main theorem of this paper. It is [2, Lemma 4.43].

Lemma 1.4. Let $A$ be an $R$-module with minimal generating set $\left\{a_{1}, \ldots, a_{n}\right\}$ in the sense that no $a_{i}$ may be deleted leaving a generating set. If $F$ is free on $\left\{x_{1}, \ldots, x_{n}\right\}, \phi: F \longrightarrow A$ is defined by $\phi\left(x_{i}\right)=a_{i}$, then $\operatorname{Ker} \phi \subseteq \mathfrak{m} F$. 
Definition 1.5. A ring is hereditary if every ideal is projective.

The following result is [2, Corollary 4.18].

Lemma 1.6. Every submodule of a projective $R$-module is projective.

In [1, Theorem 2.4], it is proved that any bounded below chain complex $Y$ in $\mathbf{C h}(R)$ whose homology modules $H_{i}(Y)$ are of finite type has a minimal projective resolution when $R$ is a commutative noetherian local ring.

We think it is not well known and we include its proof for the reader's convenience and for comparison with our result.

Theorem 1.7. Let $Y$ be a bounded below chain complex of finite type in $\mathbf{C h}(R)$. Then there is a minimal projective chain complex $G$ in $\mathbf{C h}(R)$ and a q-isomorphism $f: G \longrightarrow Y$.

Proof. We prove this theorem using induction. If $i_{0}$ is such that $H_{i}(Y)=0$ for $i \leq i_{0}$, then we can let $F_{i}=0$ for $i \leq i_{0}$ and the zero map in degrees $\leq i_{0}$ from $F$ to $Y$ is obviously a $q$-isomorphism.

Now assume that we have defined finitely generated free $R$-modules $F_{i}$ and maps $f_{i}$ for $i \leq n$ such that the following diagram

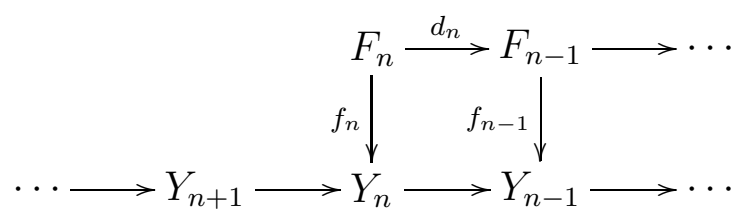

is commutative and

(a) $H_{i}(f): H_{i}(F) \longrightarrow H_{i}(Y)$ is an isomorphism for $i<n$.

(b) $g: \operatorname{Ker}\left(d_{n}\right) \longrightarrow H_{n}(Y)$ is surjective.

Now we construct $F_{n+1}$ and $f_{n+1}$ such that (a) and (b) hold for $n+1$. First note that $\operatorname{Ker}(g)$ maps to $B_{n}(Y)$ since we have the following commutative diagram with exact rows.

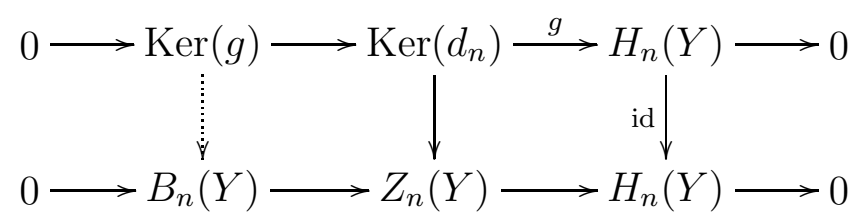

We have that the $\operatorname{Ker}(g)$ is finitely generated since $R$ is noetherian. Let $\left\{y_{1}, \ldots, y_{n}\right\}$ be a generating set of $\operatorname{Ker}(g)$. Assume that $E_{1}$ is free, hence 
projective, on $\left\{x_{1}, \ldots, x_{n}\right\}$. Define $\psi: E_{1} \longrightarrow \operatorname{Ker}(g)$ by $\psi\left(x_{i}\right)=y_{i}$. Therefore, we have the following commutative diagram where the map $h_{1}$ exists since $E_{1}$ is free.

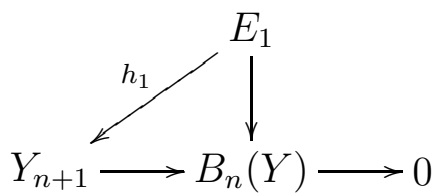

That is, the following diagram

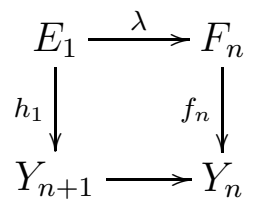

is commutative where the map $\lambda$ is the composite of $\psi: E_{1} \longrightarrow \operatorname{Ker}(g)$ with the inclusion map $\operatorname{Ker}(g) \longrightarrow F_{n}$. We have that $H_{n+1}(Y)$ is finitely generated. Let $\left\{z_{1}, \ldots, z_{m}\right\}$ be a generating set of $H_{n+1}(Y)$. Assume that $E_{2}$ is free on $\left\{t_{1}, \ldots, t_{m}\right\}$. Define $\phi: E_{2} \longrightarrow H_{n+1}(Y)$ by $\phi\left(t_{i}\right)=z_{i}$. Then we have the following commutative diagram

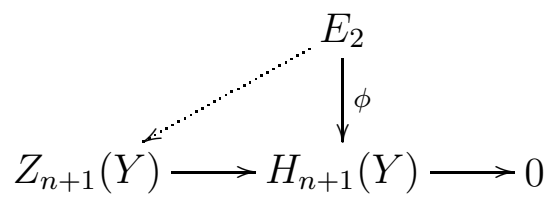

where the dotted arrow exists since $E_{2}$ is free. Let the map $h_{2}: E_{2} \longrightarrow Y_{n+1}$ be the composite of the map $E_{2} \longrightarrow Z_{n+1}(Y)$ with the inclusion map $Z_{n+1}(Y) \longrightarrow$ $Y_{n+1}$. Map $E_{2}$ to zero in $F_{n}$. Let $F_{n+1}=E_{1} \oplus E_{2}$ and $f_{n+1}=h_{1}+h_{2}$. The differential $d_{n+1}=\lambda+0$. Then we can see that the following diagram

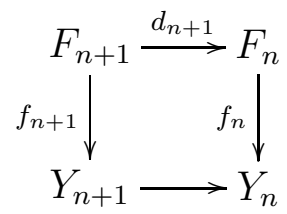

is commutative. By construction, it is clear that $H_{n}(f): H_{n}(F) \longrightarrow H_{n}(Y)$ is an isomorphism and $\operatorname{Ker}\left(d_{n+1}\right) \longrightarrow H_{n+1}(Y)$ is surjective. Hence, we have constructed a projective resolution of $Y$.

This gives some projective resolution of $Y$ and to get a minimal one, we can proceed as follows. We show that $F$ is a sum of a minimal chain complex $G$ and an exact chain complex $H$ of projective modules. Then $G$ is a minimal projective resolution of $Y$. If $F$ is not minimal, then the matrix $\left(a_{i j}\right)$ defining 
$d_{n}: F_{n} \longrightarrow F_{n-1}$ must have a unit element since $R$ is local. We can transform $\left(a_{i j}\right)$ by a finite number of elementary row and column operations to the following form

$$
\left[\begin{array}{cccc}
1 & 0 & \cdots & 0 \\
0 & & & \\
\vdots & & \left(a_{i j}^{\prime}\right) & \\
0 & & &
\end{array}\right]
$$

This means that we have a diagram

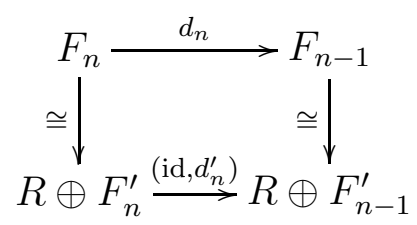

and the chain complex $F$ is the direct sum of

$$
\cdots \longrightarrow F_{n+1} \longrightarrow F_{n}^{\prime} \longrightarrow F_{n-1}^{\prime} \longrightarrow F_{n-2} \longrightarrow \cdots
$$

and

$$
\cdots \longrightarrow 0 \longrightarrow R \stackrel{\text { id }}{\longrightarrow} R \longrightarrow 0 \longrightarrow \cdots
$$

This process can be continued until we are left with a minimal projective resolution $G$ of $Y$ and the sum of pieces of the form

$$
\cdots \longrightarrow 0 \longrightarrow R \stackrel{\text { id }}{\longrightarrow} R \longrightarrow 0 \longrightarrow \cdots
$$

in various degrees. Putting these latter pieces together gives $H$ which is an exact chain complex of projective modules. Hence, there is a minimal projective chain complex $G$ in $\mathbf{C h}(R)$ and a $q$-isomorphism $f: G \longrightarrow Y$.

\section{Main Theorem}

In this section, we prove the main result of this paper.

Theorem 2.1. Let $Y$ be unbounded chain complex of finite type in $\mathbf{C h}(R)$. Then there is a minimal projective chain complex $Q$ in $\mathbf{C h}(R)$ and a q-isomorphism $g: Q \longrightarrow Y$.

Proof. Fix $n$. We have that $H_{n}(Y)$ is finitely generated. Let $\left\{y_{1}, \ldots, y_{m}\right\}$ be a minimal generating set of $H_{n}(Y)$. Assume that $E_{n, 0}$ is free, hence projective, on $\left\{x_{1}, \ldots, x_{m}\right\}$. If we define $\psi_{n, 0}: E_{n, 0} \longrightarrow H_{n}(Y)$ by $\psi_{n, 0}\left(x_{i}\right)=y_{i}$, then 
$\operatorname{Ker} \psi_{n, 0} \subseteq \mathfrak{m} E_{n, 0}$ by Lemma 1.4. Let $\operatorname{Ker} \psi_{n, 0}=E_{n, 1}$. We have $E_{n, 1}$ is projective by Lemma 1.6 since $R$ is hereditary. We get an exact sequence

$$
0 \longrightarrow E_{n, 1} \stackrel{d_{n, 1}}{\longrightarrow} E_{n, 0} \stackrel{\psi_{n, 0}}{\longrightarrow} H_{n}(Y) \longrightarrow 0
$$

where $d_{n, 1}$ is inclusion. Now consider the following chain complex

$$
F^{n}:=0 \longrightarrow E_{n, 1} \stackrel{d_{n, 1}}{\longrightarrow} E_{n, 0} \longrightarrow 0
$$

where

$$
\left(F^{n}\right)_{i}= \begin{cases}E_{n, 0} & \text { if } i=n \\ E_{n, 1} & \text { if } i=n+1 \\ 0 & \text { otherwise. }\end{cases}
$$

We easily can see that $F^{n}$ is a minimal chain complex and

$$
H_{i}\left(F^{n}\right)= \begin{cases}H_{n}(Y) & \text { if } i=n \\ 0 & \text { if } i \neq n\end{cases}
$$

Note that we have the following commutative diagram with exact rows.

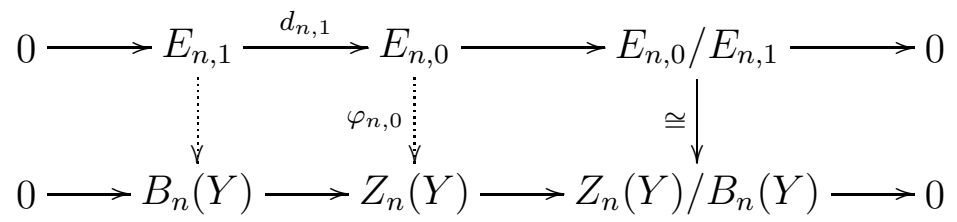

$\varphi_{n, 0}$ exists since $E_{n, 0}$ is projective and the left vertical arrow exists since the rows are exact.

Thus, we have the following commutative diagram

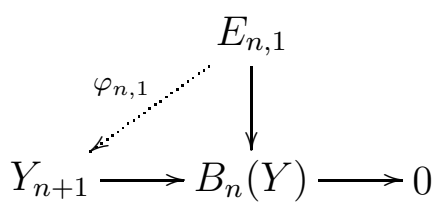

where $\varphi_{n, 1}$ exists since $E_{n, 1}$ is projective. Therefore, we have the following commutative diagram

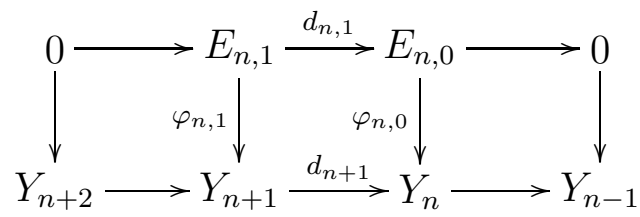


Denote the chain map between $F^{n}$ and $Y$ by $f^{n}$. Now define $Q=\underset{n}{\bigoplus_{n}} F^{n}$ and $g=\bigoplus_{n} f^{n}$. Therefore,

$$
H_{i}(Q) \cong \underset{n}{\oplus} H_{i}\left(F^{n}\right)=H_{i}\left(F^{i}\right) \cong H_{i}(Y)
$$

We can see that $Q$ is projective chain complex since for each $n$,

$$
Q_{n}=E_{n, 0} \oplus E_{n-1,1}
$$

Note that we have $d\left(Q_{n}\right) \subseteq \mathfrak{m} Q_{n-1}$. Therefore, $Q$ is a minimal chain complex. Hence, there exists a minimal projective chain complex $Q$ and a $q$-isomorphism $g: Q \longrightarrow Y$.

\section{References}

[1] P. Roberts. Homological invariants of modules over commutative rings, volume 72 of Séminaire de Mathématiques Supérieures [Seminar on Higher Mathematics]. Presses de l'Université de Montréal, Montreal, Que., 1980.

[2] J.J. Rotman. An introduction to homological algebra, volume 85 of Pure and Applied Mathematics. Academic Press Inc., New York, 1979.

Received: June 28, 2007 